\title{
Existence of non-constant positive stationary solutions of the shadow predator-prey systems with Allee effect
}

\section{Zhenhua Bao ${ }^{1 *}$ and He Liu²}

"Correspondence:

zhhbao@126.com

'School of Mathematics, Liaoning

Normal University, Dalian, 116029

China

Full list of author information is

available at the end of the article

\begin{abstract}
In this paper, we consider the dynamics of the shadow system of a kind of homogeneous diffusive predator-prey system with a strong Allee effect in prey. We mainly use the time-mapping methods to prove the existence and non-existence of the non-constant positive stationary solutions of the system in the one dimensional spatial domain. The problem is assumed to be subject to homogeneous Neumann boundary conditions.

MSC: 35K57; 35B09

Keywords: homogeneous diffusive predator-prey system; strong Allee effect in prey; shadow system; non-constant positive stationary solutions
\end{abstract}

\section{Introduction}

In this paper, we are mainly concerned with the following homogeneous diffusive predator-prey system with a strong Allee effect in prey:

$$
\begin{cases}\frac{\partial u}{\partial t}-d_{1} u_{x x}=u(1-u)\left(\frac{u}{b}-1\right)-\frac{m u v}{a+u}, & t>0, x \in(0, h \pi), \\ \frac{\partial v}{\partial t}-d_{2} v_{x x}=-d v+\frac{m u v}{a+u}, & t>0, x \in(0, h \pi), \\ u_{x}(x, t)=u_{x}(x, t)=v_{x}(x, t)=v_{x}(x, t)=0, & t>0, x=0, h \pi, \\ u(x, 0)=u_{0}(x) \geq 0, v(x, 0)=v_{0}(x) \geq 0, & x \in(0, h \pi) .\end{cases}
$$

Here $u=u(x, t)$ and $v=v(x, t)$ stand for the densities of the prey and predator at time $t>0$ and a spatial position $x \in(0, h \pi)$ with $h \in(0, \infty)$, respectively; $d_{1}, d_{2}>0$ are the diffusion coefficients of the species; $d$ is the death rate of the predator, $a$ measures the saturation effect, $m$ is the strength of the interaction. The Allee threshold $b$ is assumed to be smaller than 1 . The strong Allee effect introduces a population threshold, and the population must surpass this threshold to grow. The boundary condition here is assumed to be homogeneous Neumann type, which implies that there is no flux for the populations on the boundary. For more details on the problem (1.1), we refer interested readers to [1-7] and references therein.

In [8], the authors considered the traveling wave solutions of system (1.1). More precisely, they showed that there is a non-negative traveling wave solution of system (1.1) connecting the semi-trivial solution $(b, 0)$ and the positive equilibrium solution $\left(u_{*}, v_{*}\right)$. They also

C2014 Bao and Liu; licensee Springer. This is an Open Access article distributed under the terms of the Creative Commons Attribution License (http://creativecommons.org/licenses/by/2.0), which permits unrestricted use, distribution, and reproduction in any medium, provided the original work is properly cited. 
proved that, under certain suitable conditions, there is a small traveling wave train solution of system (1.1).

In [7], the authors considered the non-existence of non-constant positive steady state solutions, and bifurcations of spatially homogeneous and non-homogeneous periodic solutions as well as non-constant steady state solutions are studied. These results allow for the phenomenon that the rich impact of the Allee effect essentially increases the system spatiotemporal complexity.

Although the existence and non-existence of non-constant steady state solutions of the system (1.1) has been considered in [7] for finite diffusion coefficients, no results have been reported to consider the existence and non-existence of the positive non-constant steady state solutions for the shadow system corresponding to the system (1.1). The shadow system we mentioned here stands for the system where one of the diffusion coefficients tends to infinity. The readers are referred to [9-11] for the earlier contributions on the shadow systems.

Thus, the purpose of this paper is to consider the existence and non-existence of positive non-constant solutions of the following elliptic equations:

$$
\begin{cases}-d_{1} u_{x x}=u(1-u)\left(\frac{u}{b}-1\right)-\frac{m u v}{a+u}, & x \in(0, h \pi), \\ -d_{2} v_{x x}=-d v+\frac{m u v}{a+u}, & x \in(0, h \pi), \\ u_{x}(x)=u_{x}(x)=v_{x}(x)=v_{x}(x)=0, & x=0, h \pi,\end{cases}
$$

where $d_{2} \rightarrow \infty$.

The methods we used in the paper are standard time-mapping methods (see [12] and references therein for precise details on time-mapping methods). We hope that the results in the paper will allow for a clearer understanding of the rich dynamics of this particular pattern formation system. In Section 2, we state the derivation of the shadow system of the original reaction-diffusion system (1.1). In Section 3, we study the existence of the non-constant stationary solutions of the shadow system; in Section 4, we end up our discussions by drawing some conclusions.

\section{Derivation of the shadow system}

Firstly, we state the following useful a priori estimate for the non-negative solutions of system (1.1) obtained in [7]:

Lemma 2.1 Suppose that $d_{1}, d_{2}, a, b, d, m, h>0$, and that $(u(x), v(x))$ is a non-negative steady state solution of (1.1). Then either $(u, v)$ is one of constant solutions: $(0,0)$, or $(b, 0)$, or for $x \in[0, h \pi],(u(x), v(x))$ satisfies

$$
0<u(x)<1 \quad \text { and } \quad 0<v(x)<\frac{(1-b)^{2}}{4 b d}+\frac{d_{1}}{d_{2}} .
$$

For later use in our discussion, we rewrite the second equation of system (1.2) in the following way:

$$
v_{x x}+d_{2}^{-1}\left(\frac{m u v}{a+u}-d v\right)=0
$$


Lemma 2.1 implies that the non-negative solutions of the system (1.2) is bounded. Then we know that $v_{x x} \rightarrow 0$ as $d_{2} \rightarrow \infty$. Since our problem is of Neumann boundary condition type, $v$ is a constant, say $\rho$. As $d_{2} \rightarrow \infty$, there exists a positive number $C=C\left(d_{1}\right)>0$, such that

$$
\frac{(1-b)^{2}}{4 b d}+\frac{d_{1}}{d_{2}}<\frac{(1-b)^{2}}{4 b d}+C\left(d_{1}\right)
$$

Thus,

$$
0<\rho<\frac{(1-b)^{2}}{4 b d}+C\left(d_{1}\right)
$$

Integrating the second equation from 0 to $\ell \pi$, we obtain

$$
\int_{0}^{\ell \pi}\left(\frac{m u}{a+u}-d\right) \rho d x=0
$$

Thus, system (1.2) reduces to the following single $\rho$-parameterized scalar reactiondiffusion equation:

$$
\left\{\begin{array}{l}
-d_{1} u_{x x}=u(1-u)\left(\frac{u}{b}-1\right)-\frac{m \rho u}{a+u} \\
u^{\prime}(0)=u^{\prime}(h \pi)=0
\end{array}\right.
$$

subject to the additional condition (2.3) and the condition

$$
0<u, \rho<C_{1}:=\max \left\{1, \frac{(1-b)^{2}}{4 b d}+C\left(d_{1}\right)\right\}
$$

\section{Existence of non-constant positive stationary solutions of the shadow system}

In this section, we mainly concentrate on the existence of the non-constant positive solutions of the reduced shadow system (2.4).

For the purpose of our investigations, we define

$$
f(u)=u(1-u)\left(\frac{u}{b}-1\right)-\frac{m \rho u}{a+u} \text { and } \quad F(u)=\int_{0}^{u} f(s) d s .
$$

Then we introduce the following energy functional:

$$
E(x)=\frac{d_{1}}{2}\left(u_{x}(x)\right)^{2}+F(u(x))
$$

From (3.2), we can find that, for any $x \in(0, h \pi), E^{\prime}(x) \equiv 0$, and $F(u(x))<F(\beta)=F(\tau)$, where $\beta:=u(0)$, and $\tau:=u(h \pi)$.

It follows that if $u=u(x)$ is a solution of (2.4), then $F(u)$ must attain its local minimal value at a point in $(\beta, \tau)$.

We rewrite $f(u)$ as

$$
f(u)=\frac{m u}{a+u}(\ell(u)-\rho),
$$


where

$$
\ell(u):=\frac{(a+u)(1-u)(u-b)}{k b} .
$$

We have the following lemma on the properties of the function $\ell(u)$ defined above.

Lemma 3.1 For any $u \in(0, b) \cup(1, \infty)$, we have $\ell(u)<0$, while $\ell(u)>0$ for any $u \in(b, 1)$. In particular, $\ell(b)=\ell(1)=0$. Moreover,

1. Suppose that $a b+a-b<0$ holds. Then for any $u \in\left(\lambda_{*}, \lambda^{*}\right), \ell(u)$ is increasing, while for any $u \in\left(0, \lambda_{*}\right) \cup\left(\lambda^{*}, \infty\right), \ell(u)$ is decreasing, where

$$
\begin{aligned}
& \lambda^{*}:=\frac{b+1-a+\sqrt{(b+1-a)^{2}+3(a b+a-b)}}{3}, \\
& \lambda_{*}:=\frac{b+1-a-\sqrt{(b+1-a)^{2}+3(a b+a-b)}}{3} .
\end{aligned}
$$

2. Suppose that $a b+a-b \geq 0$ holds. Then, for any $u \in\left(0, \lambda^{*}\right), \ell(u)$ is increasing, while for any $u \in\left(\lambda^{*}, \infty\right), \ell(u)$ is decreasing.

Proof It is obvious that for any $u \in(0, b) \cup(1, \infty)$, we have $\ell(u)<0$, while $\ell(u)>0$ for any $u \in(b, 1)$. We can directly check that

$$
\begin{aligned}
\ell^{\prime}(u) & =\frac{-3 u^{2}-2(a-b-1) u+(a b+a-b)}{k b} \\
& =-\frac{3}{k b}\left(u-\lambda_{*}\right)\left(u-\lambda^{*}\right) .
\end{aligned}
$$

Because $\lambda^{*}>\lambda_{*}>0$ provided that $a b+a-b<0$, we conclude from (3.5) that $\ell(u)$ is increasing $\left(\ell^{\prime}(u)>0\right)$ for $u \in\left(\lambda_{*}, \lambda^{*}\right)$, while for any $u \in\left(0, \lambda_{*}\right) \cup\left(\lambda^{*}, \infty\right), \ell(u)$ is decreasing $\left(\ell^{\prime}(u)<0\right)$. The second part of the lemma can be proved similarly.

From Lemma 3.1, it follows that $\ell(u)$ attains its maximum value $\ell_{*}:=\ell\left(\lambda^{*}\right)$ at $u=\lambda^{*}$. If $\rho>\ell_{*}$ holds, then $f(u)<0$ for all $u \in(0, \infty)$. Thus, $F(u)$ does not has its minimal value point in $(0, \infty)$, which implies that the shadow system (2.4) does not possess positive nonconstant stationary solutions. Similarly, if $\rho=\ell_{*}$ holds, then system (2.4) does not also possess positive non-constant stationary solutions.

Thus, in order for the shadow system to have non-constant positive stationary solutions, we need to concentrate on the case when $\rho \in\left(0, \ell_{*}\right)$.

In this case, there exist two zeros of $f(u)=0$, and we can denote them by

$$
u_{ \pm}(\rho)=\frac{k-1 \pm \sqrt{(k+1)^{2}-4 k m \mu}}{2}
$$

with $b<u_{-}(\rho)<\lambda^{*}<u_{+}(\rho)<1$.

Since $f(u)<0$ for $0<u<u_{-}(\rho), f(u)>0$ for $u_{-}(\rho)<u<u_{+}(\rho)$, it follows that $F(u)$ is convex in $\left(0, u_{+}(\rho)\right)$, and concave in $\left(u_{-}(\rho), \infty\right)$, and $F(u)$ taking its local minimum value at $u=u_{-}(\rho)$. In other words, $F(u)$ is decreasing in $\left(0, u_{-}(\rho)\right) \cup\left(u_{+}(\rho), \infty\right)$, and increasing in $\left(u_{-}(\rho), u_{+}(\rho)\right)$. 
Thus, the problem admits solutions for some $h>0$ if and only if $\rho \in\left(0, \ell_{*}\right)$ and we are now deriving the precise information on the suitable $h>0$ such that the problem has positive non-constant stationary solutions.

If $F(0) \geq F\left(u_{+}(\rho)\right)$ holds, then there exists a unique $\beta^{*} \in\left(0, u_{-}(\rho)\right)$, such that $F\left(\beta^{*}\right)=$ $F\left(u_{+}(\rho)\right)$. Define

$$
\beta_{0}:= \begin{cases}0, & \text { if } F(0) \leq F\left(u_{+}(\rho)\right) \\ \beta^{*}, & \text { if } F(0) \geq F\left(u_{+}(\rho)\right)\end{cases}
$$

Then for any $\beta \in\left(\beta_{0}, u_{-}(\rho)\right)$, there exists a unique $\beta^{* *} \in\left(u_{-}(\rho), u_{+}(\rho)\right)$, such that $F\left(\beta_{0}\right)=$ $F\left(\beta^{* *}\right)$.

By the definition of $E(x)=d_{1}\left(u_{x}(x)\right)^{2} / 2+F(u(x))$, and the fact that $E^{\prime}(x) \equiv 0$, we have

$$
u_{x}(x)=\sqrt{2(F(\beta)-F(u)) / d_{1}}>0, \quad x \in(0, h \pi) .
$$

Fix $u(h \pi)=\tau$, and integrate (3.8); we have

$$
h(\beta)=\frac{\sqrt{d_{1}}}{\pi} \int_{\beta}^{\tau} \frac{d u}{\sqrt{2(F(\beta)-F(u))}} .
$$

In the following, we want to show the limit of $h(\beta)$ as $\beta \rightarrow u_{-}(\rho)$. In fact, following the same argument in [12] (say, for example, pp.314-316), one can verify that

$$
\lim _{\beta \rightarrow u_{-}(\rho)} h(\beta)=\frac{\sqrt{d_{1}}}{\pi \sqrt{f^{\prime}\left(u_{-}(\rho)\right)}} .
$$

Also following [12], for a given number $u \in(\beta, \tau)$, we define $u=g(s)$ by the relation

$$
F(g(s))-F\left(u_{-}(\rho)\right)=\frac{s^{2}}{2}, \quad \operatorname{sign} s=\operatorname{sign}\left(u-u_{-}(\rho)\right)=\operatorname{sign}(f(u)) .
$$

Then $s=g^{-1}(u)$ is well defined and is strictly increasing in $(\beta, \tau)$, since in this interval $F(u)$ is convex and takes a strict minimum at $u=u_{-}(\rho)$.

Let $p>0$ be given by

$$
\frac{1}{2} p^{2}=F(\beta)-F\left(u_{-}(\rho)\right)>0 .
$$

Then we have

$$
h(\beta)=\int_{-p}^{p} \frac{g^{\prime}(s) d s}{\sqrt{p^{2}-s^{2}}}=\int_{0}^{\pi} g^{\prime}(-p \cos t) d t,
$$

where we make another change of variable, $s=-p \cos t, 0 \leq t \leq \pi$. 
By the same argument as on p.314 of [12], we have

$$
\begin{aligned}
& g^{\prime}(s)=\frac{\sqrt{2 \bar{F}(u)}}{|f(u)|}, \quad \bar{F}(u)=F(u)-F\left(u_{-}(\rho)\right), \\
& g^{\prime \prime}(s)=-\frac{f^{2}-2 f^{\prime} \bar{F}}{f^{3}}(u), \quad g^{\prime \prime}(0)=-\frac{f^{\prime \prime}}{3 f^{\prime 2}}\left(u_{-}(\rho)\right), \\
& g^{\prime \prime \prime}(s)=-\frac{g^{\prime}(s)}{f^{4}(u)} H(u), \\
& g^{\prime \prime \prime}(0)=\frac{1}{12\left(f^{\prime}\left(u_{-}(\rho)\right)\right)^{7 / 2}}\left[5\left(f^{\prime \prime}\right)^{2}-3 f^{\prime} f^{\prime \prime \prime}\right]\left(u_{-}(\rho)\right),
\end{aligned}
$$

where

$$
H(u):=2 f(u) f^{\prime \prime}(u) \bar{F}(u)+3 f^{\prime}(u)\left[f^{2}(u)-2 f^{\prime}(u) \bar{F}(u)\right]
$$

By (3.1), we have

$$
f(u)=u(1-u)\left(\frac{u}{b}-1\right)-m \rho \frac{u}{a+u} .
$$

Then after direct calculations, we have

$$
\begin{aligned}
& f^{\prime}(u):=-\frac{3 u^{2}}{b}+2\left(\frac{1}{b}+1\right) u-1-m \rho a \frac{1}{(a+u)^{2}} \\
& f^{\prime \prime}(u):=-\frac{6}{b} u+2\left(\frac{1}{b}+1\right)+\frac{2 m \rho a}{(a+u)^{3}} \\
& f^{\prime \prime \prime}(u):=-\frac{6}{b}-\frac{6 m \rho a}{(a+u)^{4}}
\end{aligned}
$$

Clearly, the function $f^{\prime \prime}(u)=0$ has a unique root $u_{1}>0$ since $f^{\prime \prime}(0)>0, f^{\prime \prime \prime}(0)<0$ and $\lim _{u \rightarrow+\infty} f^{\prime \prime}(u)=-\infty$.

On the other hand, by the properties of $f$, we know that $f$ has two critical points in $\left(0, u_{-}(\rho)\right)$, denoted by $c_{1}$ and $c_{2}$, that is to say, $f^{\prime}\left(c_{1}\right)=f^{\prime}\left(c_{2}\right)=0$. Since $f^{\prime}(0)<0$, $\lim _{x \rightarrow \pm \infty} f^{\prime}(u)=-\infty$, and $f^{\prime}(u)$ has a unique positive critical point $u_{1}>0$, it follows that $c_{1}<u_{1}<u_{2}$.

Thus, the results in Lemma 4.4 in [12] hold true in our problem. That is, for any $u \in$ $\left(0, u_{-}(\rho)\right)$, we have $H(u)<0$, which together with (3.14) implies that $g^{\prime \prime \prime}(s)>0$.

By (3.12), we have $d p / d \beta<0$. By (3.13), we have

$$
h^{\prime}(p)=-\int_{0}^{\pi} \cos \operatorname{tg}^{\prime \prime}(s) d t, \quad h^{\prime \prime}(p)=\int_{0}^{\pi} \cos ^{2} \operatorname{tg}^{\prime \prime \prime}(s) d t, \quad s=-p \cos t .
$$

Since

$$
h^{\prime}(0)=\frac{d h(0)}{d p}=-g^{\prime \prime}(0) \int_{0}^{\pi} \cos t d t=0,
$$

and $h^{\prime \prime}(p)>0$ due to $g^{\prime \prime \prime}(s)>0$, it follows that $h^{\prime}(p)>0$ or equivalently $d h / d p>0$. This together with the fact that $d p / d \beta<0$, we can conclude that $d h / d \beta<0$. 
Summarizing the analysis above, we can conclude the following.

Theorem 3.2 Let $\ell(u), \lambda^{*}$ be defined in (3.3) and (3.4), and $u_{-}(\rho)$ be defined in (3.6). Then the shadow system (2.4) with the condition (2.3) has no non-constant positive stationary solutions if $h \geq \ell_{*}:=\ell\left(\lambda^{*}\right)$, and has non-constant positive stationary solutions if and only if $\rho \in\left(0, \min \left\{\ell_{*}, C_{1}\right\}\right)$ and

$$
h>h_{0}:=\frac{\pi}{f^{\prime}\left(u_{-}(\rho)\right)} \text { and } d=\int_{0}^{h \pi} \frac{m u}{a+u} d x .
$$

\section{Conclusions}

In this paper, we studied the existence and non-existence of the positive non-constant stationary solutions of a shadow system corresponding to a kind of diffusive homogeneous predator-prey system with Holling type-II functional response and strong Allee effect in prey. We hope that the results in the paper will allow for the clearer understanding of the rich dynamics of this particular pattern formation system. Future work might include considering the qualitative behavior of the parabolic shadow system.

Competing interests

The authors declare that they have no competing interests.

Authors' contributions

All authors have equal contributions. All authors read and approved the final manuscript.

\section{Author details}

'School of Mathematics, Liaoning Normal University, Dalian, 116029, China. ${ }^{2}$ School of Mathematics, Physics and Biological Engineering, Inner Mongolia University of Science and Technology, Baotou, 014010, China.

\section{Acknowledgements}

The authors are very grateful to the anonymous referee for his/her valuable comments and suggestions, which led to an improved presentation of the manuscript.

Received: 23 April 2014 Accepted: 30 July 2014 Published: 15 Aug 2014

\section{References}

1. Boukal, DS, Sabelis, MW, Berec, L: How predator functional responses and Allee effects in prey affect the paradox of enrichment and population collapse. Theor. Popul. Biol. 72, 136-147 (2007)

2. Holling, CS: The components of predation as revealed by a study of small mammal predation of the Euorpean pine swalfy. Can. Entomol. 91, 293-320 (1959)

3. Lewis, M, Karevia, P: Allee dynamics and the spread of invading organisms. Theor. Popul. Biol. 43, 141-158 (1993)

4. Morozov, A, Petrovovskii, S, Li, B: Spatiotemporal complexity of patchy invasion in a predator-prey system with the Allee effect. J. Theor. Biol. 238, 18-35 (2006)

5. Nisbet, RM, Gurney, WSC: Modelling Fluctuating Populations. Wiley, New York (1982)

6. Petrovovskii, S, Morozov, A, Li, B: Regimes of biological invasion in a predator-prey system with the Allee effect. Bull. Math. Biol. 67, 637-661 (2005)

7. Wang, J, Shi, J, Wei, J: Dynamics and pattern formation in a diffusive predatory-prey system with strong Allee effect in prey. J. Differ. Equ. 251, 1276-1304 (2011)

8. Hsu, C, Yang, C, Yang, T, Yang, T: Existence of traveling wave solutions for diffusive predator-prey type systems. J. Differ. Equ. 252, 3040-3075 (2012)

9. Nishiura, Y: Global structure of bifurcating solutions of some reaction-diffusion systems. SIAM J. Math. Anal. 13, 555-593 (1982)

10. Opial, Z: Sur les périodes des solutions de l'équation différentielle $x^{\prime \prime}+g(x)=0$. Ann. Pol. Math. 10, 49-72 (1961)

11. Keener, J: Activators and inhibitors in pattern formation. Stud. Appl. Math. 59, 1-23 (1978)

12. Jang, J, Ni, W, Tang, M: Global bifurcation and structure of Turing patterns in the 1-D Lengyel-Epstein model. J. Dyn. Differ. Equ. 16, 297-320 (2004)

10.1186/1687-1847-2014-226

Cite this article as: Bao and Liu: Existence of non-constant positive stationary solutions of the shadow predator-prey systems with Allee effect. Advances in Difference Equations 2014, 2014:226 